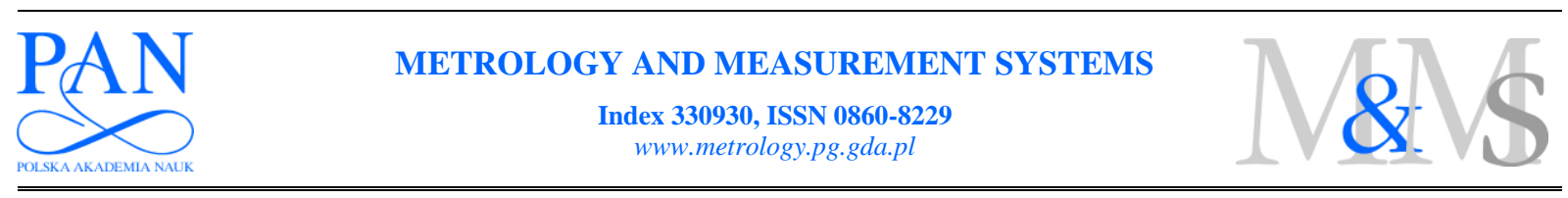

\title{
GENERATION OF A ZONE CHIRP IN UNIFORM BRAGG GRATING AS A WAY OF OBTAINING DOUBLE FUNCTIONALITY OF A SENSOR
}

\section{Piotr Kisala}

Lublin University of Technology, Institute of Electronics and Information Technology, Nadbystrzycka 38A, 20-618 Lublin, Poland (هp.kisala@pollub.pl, +48 81538 4317)

\begin{abstract}
This paper presents a method of using a sensor with uniform Bragg grating with appropriately generated zone chirp. The presented method can be used for measuring two physical quantities, namely strain and temperature. By providing the same temperature sensitivity and different sensitivity to strain of two parts of a sensor, and experimental measurement of qualities of the proposed system and its calibration (experimental determination of sensitivity), verification of the results obtained from laboratory tests and the possibility of its practical implementation has been confirmed. The sensor grating was placed in such a way that its half was in the zone of a variable value of axial strain caused by changes of the cross-section of the sample. The other half, however, was in the zone of a constant cross-section of the sample and of constant value of strain, caused by the force stretching the sample. The obtained errors of non-linearity of processing characteristics for measuring strain and temperature of the proposed system were $2.7 \%$ and $1.5 \%$ respectively, while coefficients of sensitivity to strain and temperature were $0.77 \times 10^{-6} \mathrm{~m} / \varepsilon$ and $4.13 \times 10^{-12} \mathrm{~m} / \mathrm{K}$ respectively. The maximum differences between the values obtained from the indirect measurement and the set values were $110 \mu \varepsilon$ for strain and $3.8^{\circ} \mathrm{C}$ for temperature, for a strain of $2500 \mu \varepsilon$ and a temperature of $40^{\circ} \mathrm{C}$.
\end{abstract}

Keywords: fibre Bragg gratings, strain and temperature sensor, temperature-insensitive measurement method.

(C) 2012 Polish Academy of Sciences. All rights reserved

\section{Introduction}

For measurements of several physical quantities, the sensitivity of a Bragg grating to temperature is an undesirable feature and complicates the possibility of its use as part of instruments for measuring elements such as strain [1], pressure [2] or mass [3]. In the case of measuring the shift in the Bragg wavelength of a single grating, it is difficult to specify if and to what extent the change was caused by the strain or temperature of the grating. A common solution to this problem is using a pair of FBG mounted on opposite sides of the surface of the element undergoing bending [4]. The systems containing a pair of FBG, possess wide possibilities of use, due to high resolution of temperature measurements [5], and their sensitivity [6]. Recent studies indicate, however, that the mentioned differential systems have limitations involving checking a limited number of gratings. A simultaneous measurement of strain and temperature is possible by specifying the shift in the wavelength of two gratings in a situation when their sensitivity to the two measurands is different [7]. A common approach is to measure the strain and temperature simultaneously, using a hybrid sensor consisting of two FBGs and a Long Period Grating (LPG). The LPG has much higher temperature sensitivity than FBG but its strain sensitivity is lower. The known use of the two elements of an optoelectronic sensor, in the form of a Bragg grating inscribed on fibre (in-fibre Bragg grating - IFBG) and polarising filters [8], allows for obtaining a sensor system whose measuring part is $25 \mathrm{~cm}$ long, which can be impractical in many applications. 
Strain and temperature sensors using a connection of FBG with a fibre-optic interferometer system constitute a separate group. The solutions based on a Sagnac interferometer and LPG and/or FBG are characterised by high sensitivity and low cost of production [9]. Systems based on FBG couple [10] or on FBG connected with another element being sensitive to one of the discussed measurands [11], require pre-calibration of each element (grating and interferometer) in order to be used in practice. However, it results in complications in their application. Usually to measure strain and temperature simultaneously, two Bragg gratings are used [12,13] (usually produced by using a phase mask [14] or a Bragg grating connected with a different optoelectronic element, whose spectral characteristics is modulated by temperature and strain [15]. One of the methods of measuring strain and temperature simultaneously is to record FBG with different Bragg lengths at the same position of the optical fibre [16]. This method requires two phase masks and two broadband light sources, in order to interrogate the signal from the FBG. However, when the broadband is exposed to UV light, its mechanical strength becomes weaker. Determining the strain and temperature recovery using an FBG is an example of an indirect measurement [17]. In some cases it requires an inverse problem solution [18-20].

In this article a head of the author's design sensor - made of a single Bragg grating placed on a metal sample designed for this purpose - was used for parallel measurements of the discussed measurands (strain and temperature).

The shape of the sample and the place of application were designed to obtain different sensitivities on the same grating - for strain and sensitivity to temperature. The key element was to obtain the same temperature sensitivity and different sensitivity to strain of the sensor, as well as to check the proposed sensor system by calibrating it (experimental determination of sensitivity) and, next, to verify the results obtained during laboratory tests.

\section{Scheme of the sensor system based on uniform Bragg grating, for measurements of two physical quantities}

A sensor system with uniform Bragg grating was used for parallel measurements of strain and temperature. The sample on which the grating was applied was designed in a manner making it possible to obtain an appropriate strain gradient along axis $z$, causing the change of bandwidth of the spectrum reflected from the grating - see Fig. 1.

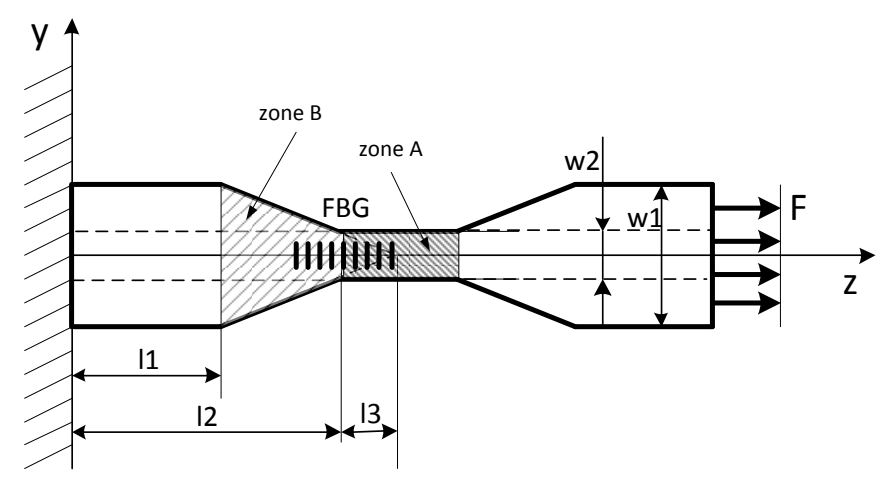

Fig. 1. Dimensions and shape of the sample used in the sensor system with a single FBG for simultaneous measurement of strain and temperature.

The values of particular characteristic quantities, marked in the above figure, are as follows: Distance 11, 12, $13-84 \mathrm{~mm}, 94 \mathrm{~mm}, 4 \mathrm{~mm}$, respectively, width in the w1 and w2 
sample zones $-25 \mathrm{~mm}$ and $6 \mathrm{~mm}$ respectively, sample thickness: $t=1 \mathrm{~mm}$. The grating was placed so that its half was in zone $\mathrm{B}$, of changeable axis strain value due to changing of the cross-section of the sample (right hatched area in Fig. 1). The other half of the grating was in zone A, of constant strain and sample width, post application of the force stretching sample $F$ (left hatched area in Fig. 1). The sample was made of steel, with known Young modulus equal to $E=20.53 \times 10^{10} \mathrm{~N} / \mathrm{m}^{2}$ and temperature expansion coefficient: $\alpha_{p}=12 \times 10^{-6} / \mathrm{K}$.

Fig. 2 presents the scheme of an experimental system for parallel measurement of strain and temperature by means of a sensor with one FBG. The light from a broadband SLED is directed by a $3 \mathrm{~dB}$ coupler onto a Bragg grating whose reflectance spectrum is registered on an optical spectrum analyser (OSA) with a resolution of $0.01 \mathrm{~nm}$. The sample together with the Bragg grating undergo a stretching force $F$ and are strained (along axis $z$ ).

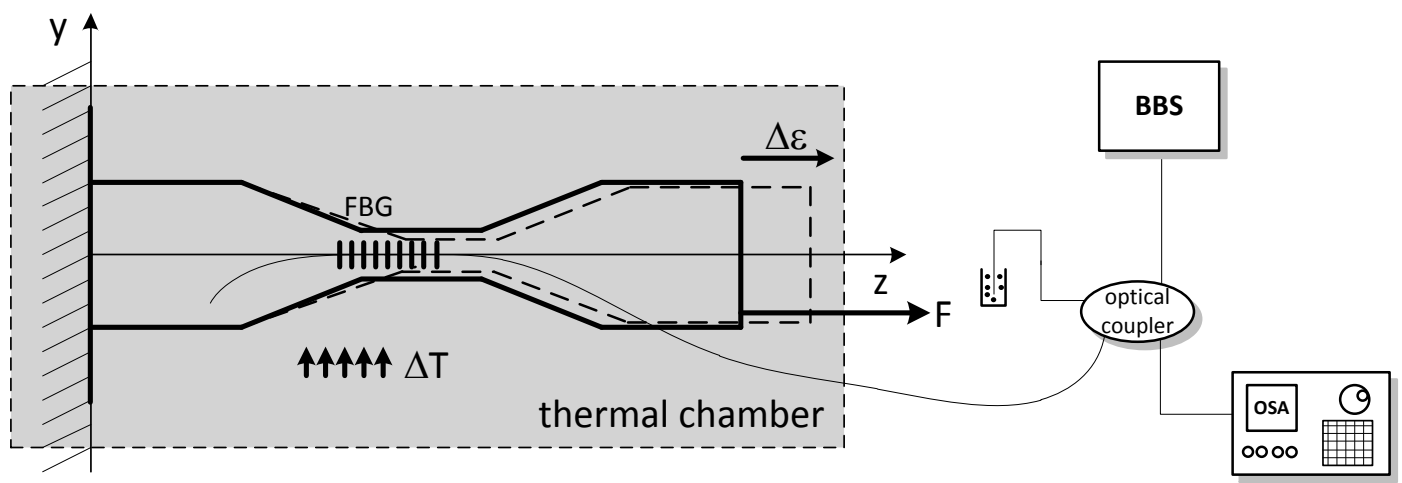

Fig. 2. Experimental system for measuring strain and temperature by means of a sensor with one FBG. BSS - broadband light source, OSA - optical spectrum analyser.

Extending the free end of the sample (the shape is marked with a dashed line) causes the creation of resonance peaks of FBG - two overlapping spectra, which result from the changeable shape of the cantilever-sample. Both reflectance spectra have the same temperature sensitivity because the thermal expansion coefficient and photo-elastic constant are the same for both parts of the same grating: the part in zone A and the part in zone B according to the equation:

$$
k_{T}=\left(\alpha_{\Lambda}+\alpha_{n}\right) K^{-1}=\left[\left(\frac{1}{\Lambda} \frac{\partial \Lambda}{\partial T}\right)+\left(\frac{1}{n_{\text {eff }}} \frac{\partial n_{\text {eff }}}{\partial T}\right)\right] K^{-1},
$$

where $\alpha_{\Lambda}$ is the thermal expansion coefficient of the broadband (for silica glass its value amounts to $\left.0.55 \times 10^{-6}\right), \alpha_{n}$ is a thermo-optical coefficient $\left(\alpha_{n}=8.6 \times 10^{-6}\right), n_{\text {eff }}$ is the effective refractive index, $T$ is temperature, $K$ denotes Kelvin and $\Lambda$ is the period of the grating.

The change of the Bragg wavelength, caused by straining for each zone, is proportional to the axial straining of the grating. Marking the Bragg wavelength of the grating part in zone A as $\lambda_{B}^{A}$ and, analogously, the Bragg wavelength of the grating part in zone $\mathrm{B}$ as $\lambda^{B}{ }_{B}$, it is possible to track developments in both zones simultaneously.

In order to perform a simultaneous measurement of changes of strain $\Delta \varepsilon$ and temperature $\Delta T$ we will use the changes of the Bragg wavelength of the grating part from zone A, (after placing the entire system in a thermal chamber with controlled temperature) - marked as $\Delta \lambda^{A}{ }_{B}$ and the changes of reflective spectrum bandwidth of the entire grating, marked as $\Delta \lambda_{\text {chirp }}$ (Fig. $3)$. 


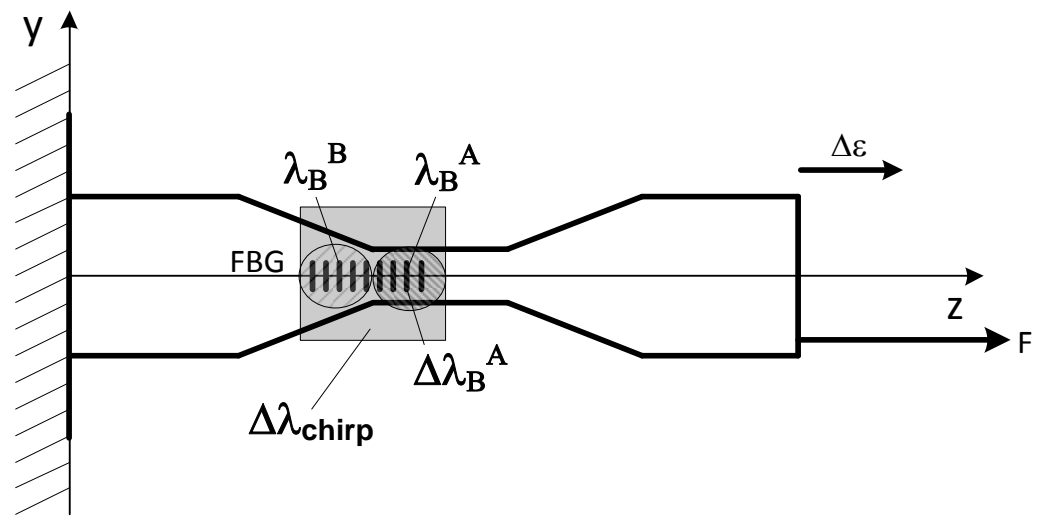

Fig. 3. Marking the wavelength and spectral width used in calculations and mathematical analysis.

For such a case, the matrix of dependence of grating parameters on changes of temperature and strain can be presented as shown below:

$$
\left[\begin{array}{c}
\Delta \lambda_{B}^{A} \\
\Delta \lambda_{\text {chirp }}
\end{array}\right]=\left[\begin{array}{cc}
K_{T \lambda A} & K_{\varepsilon \lambda A} \\
K_{T \lambda c h i r p} & K_{\varepsilon \lambda c h i r p}
\end{array}\right] \times\left[\begin{array}{c}
\Delta \varepsilon \\
\Delta T
\end{array}\right],
$$

where $K_{T \lambda A}$ and $K_{\varepsilon \lambda A}$ are displacement sensitivities of Bragg wavelength $\Delta \lambda^{A}{ }_{B}$ of the grating part placed in zone A to temperature and strain, respectively, while $K_{T \lambda c h i r p}$ and $K_{\varepsilon \lambda c h i r p}$ specify sensitivities of the width of spectral reflectance characteristics of the Bragg grating $\Delta \lambda_{\text {chirp }}$ to temperature and strain, respectively.

The change of temperature and strain will cause a change of the Bragg wavelength of the part of the grating in zone $\mathrm{B}$, as presented below:

$$
\Delta \lambda_{B}^{A}=K_{T \lambda A} \Delta T+K_{\varepsilon \lambda A} \Delta \varepsilon,
$$

where $K_{T \lambda A}$ and $K_{\varepsilon \lambda A}$ are Bragg wavelength sensitivities of the grating in zone A to temperature and strain, respectively.

Fig. 4 shows a photo of a sample with applied FBG and Fig. 5 shows the strain (plane shape) and stresses (colour) in the sample, calculated by means of finite element method (FEM).
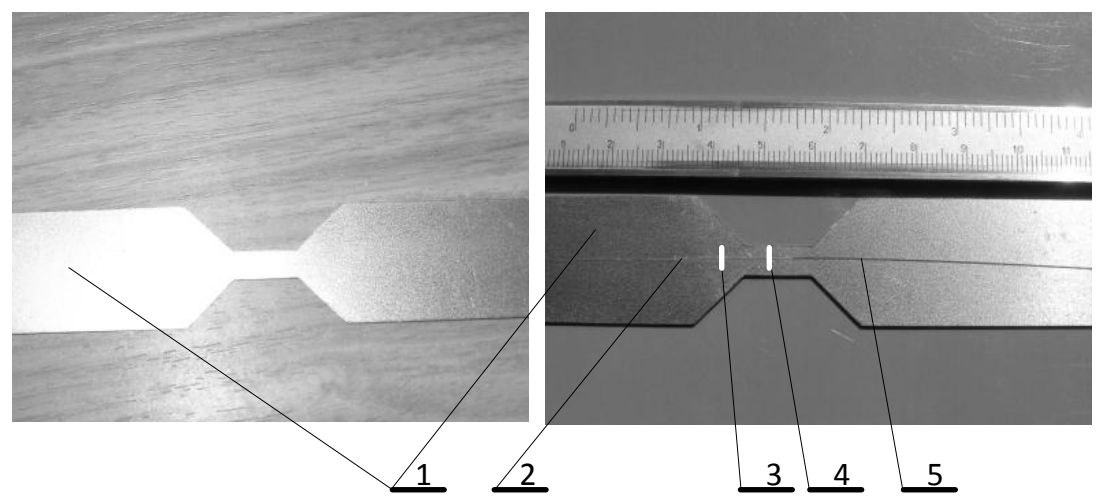

Fig. 4. The tested sensor with Fibre Bragg Grating placed on a profile sample. 1 - metal cantilever, 2. Glue joint (sikadur-30), 3-the beginning of FBG, 4 - the end of FBG, 5 - SMF28 single-mode fibre. 


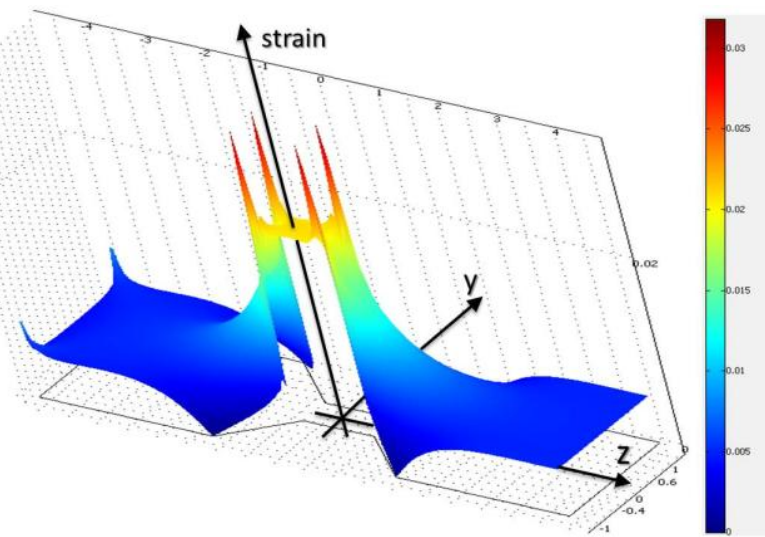

Fig. 5. Distribution of strain along axes y and $\mathrm{z}$ determined by means of FEM.

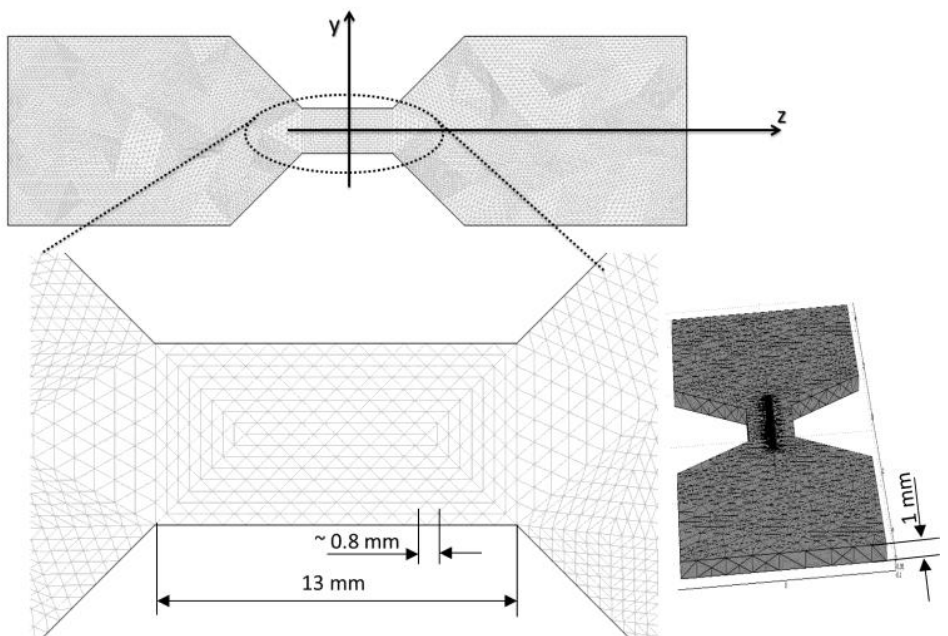

Fig. 6. Comparison of the size of sensor construction element with the size of finite elements of FEM.

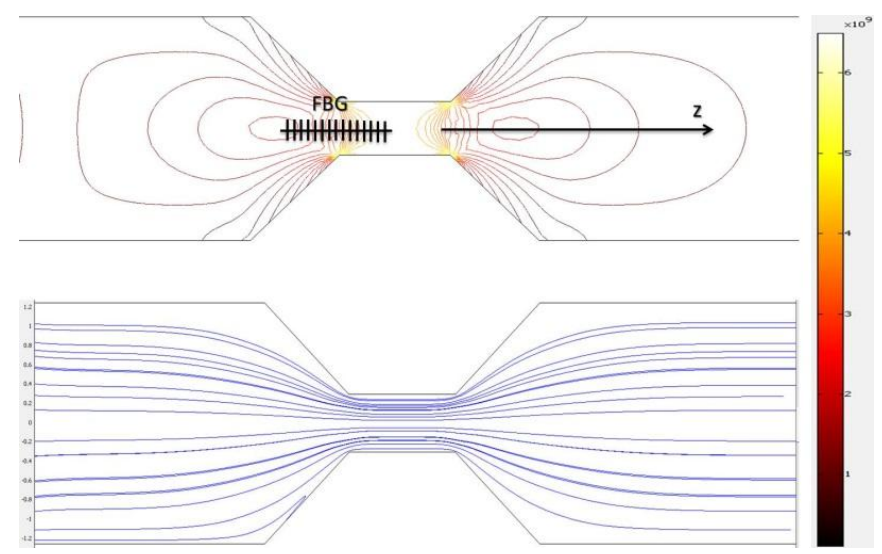

Fig. 7. Normal strain global (top) and first principal strain direction (bottom).

Comparison of the size of sensor construction element with the size of FEM finite elements is presented in Fig. 6. The accurate determination of the strain value is limited due to division of continuous physical space into a number of finite elements, resulting from the use of the FEM method. Fig. 7 shows the values of normal strain global and first principal strain direction. The figure also shows the place of application of the Bragg grating. The strain distribution in section B of the cantilever is nonuniform, as illustrated in Fig. 6. It corresponds with the normal strain global and first principal strain direction depicted in Fig. 7. 


\section{Theoretical analysis}

Rationale for selection of width of the spectral grating characteristics $\Delta \lambda_{\text {chirp }}$ as the second parameter, (for simultaneous measurement of temperature and strain) is presented below.

The resonance bandwidth depends on the value of the grating period in a way which can be presented by the equation below:

$$
\Delta \lambda_{\text {chirp }}=2 n_{\text {eff }}\left(\Lambda^{A}-\Lambda_{\min }^{B}\right),
$$

therefore it is proportional to the range of the grating period. In equation (4) $\Lambda^{A}$ and $\Lambda^{B}{ }_{\min }$ are maximum (after applying stretching force to the system, maximum grating period will be the one in zone A of the sample) and minimum period of the grating, which will experience a chirp after stretching, will be in zone B. Therefore, it is possible to determine the dependence of spectral width of the grating from extortion (applied stretching force) causing the strain of the grating. The cross-section of the stretched sample can be described as a function of position along axis $z$, as presented in the equation below:

$$
A^{B}(z)=g s^{B}(z),
$$

where $g$ is the thickness of the sample, $s^{B}(z)$ is the width in zone B, dependent on the position of the sample along axis $z$.

The width of the sample in zone B is a function of the position along axis $z$ and depends on maximum (initial) width $s_{1}$ of the sample, for the area in zone B has a trapezoidal shape, and we obtain an equation illustrating the dependence $s^{B}(z)$ :

$$
s^{B}(z)=s_{1}\left(1-\frac{z}{z_{0}}\right) .
$$

where $z_{0}$ is the point where the sample width equals zero. The distance between this point and the immoveable end of the sample is $l_{2}+l_{3}$. Taking into account Hooke's Law, the strain along axis $z$ in zone B, caused by $F$ force is as follows:

$$
\varepsilon^{B}(z)=\frac{F}{A(z) E}=\frac{F}{E \cdot s_{1}\left(1-\frac{z}{z_{0}}\right)},
$$

where $E$ is Young's modulus.

It should be noticed that uneven strain of the sample causes creation of chirp in the grating in zone $\mathrm{B}$, therefore the grating periods will be a function of position $z$. The relation between the grating period and the position along axis $z$ in zone $\mathrm{B}$ can be presented as in the equation below:

$$
\Lambda^{B}(z)=\Lambda_{0}\left(1+\varepsilon^{B}(z)\right)
$$

where $\Lambda_{0}$ is the period of unstrained grating, $\varepsilon^{B}(z)$ is the strain of the grating for a given point along axis $z$ in zone B. The created chirp of the grating period can therefore be expressed with a function of maximum $\varepsilon_{\max }$ and minimum $\varepsilon_{\min }$ grating strain:

$$
\Delta \Lambda_{\text {chirp }}=\Lambda_{0}\left(\varepsilon_{\max }-\varepsilon_{\min }\right) \text {. }
$$

Substituting (9) to (4), the obtained dependence specifies the spectral width connected with the period of the strained grating.

$$
\Delta \lambda_{\text {chirp }}=2 n_{\text {eff }} \Delta \Lambda_{\text {chirp }} .
$$

Therefore the spectral width of the grating varies linearly with the applied force: 


$$
\Delta \lambda_{\text {chirp }}=2 n_{\text {eff }} \Lambda_{0} F \frac{\frac{1}{\left(1-z / z_{0}\right)}-1}{E \cdot s_{1}} .
$$

Depending on the applied force causing the grating strain, according to (11), there is no dependence on $\Delta \lambda_{\text {chirp }}$ temperature. Taking into account the fact that the temperature sensitivity of $\Delta \lambda_{\text {chirp }}$ equals zero and presenting its dependence on strain as:

$$
\Delta \lambda_{\text {chirp }}=K_{\varepsilon \lambda c h i r p} \Delta \varepsilon,
$$

and adding (3), we obtain a reduced matrix equation of the sensor:

$$
\left[\begin{array}{c}
\Delta T \\
\Delta \varepsilon
\end{array}\right]=\frac{1}{K_{T \lambda A} K_{\varepsilon \lambda c h i r p}}\left[\begin{array}{cc}
K_{\varepsilon \lambda c h i r p} & -K_{\varepsilon \lambda A} \\
0 & K_{T \lambda A}
\end{array}\right] \times\left[\begin{array}{c}
\Delta \lambda_{B}^{A} \\
\Delta \lambda_{\text {chirp }}
\end{array}\right] .
$$

Creation of the chirp induced experimentally in a laboratory, by means of intended preparation of the measurement system with a standard (instead of tapered) FBG and in homogenous strain, allowed for obtaining information concerning measurands which were coded not only in the Bragg wavelength but also in the width of grating reflective spectrum. Analysing equation (13) we can see that the change of temperature and strain can be specified based on the shift of the Bragg wavelength and the width of spectrum of a single uniform grating. This confirms the possibility of simultaneous measurement of strain and temperature by means of a single FBG.

\section{The results of simulation and experimental tests}

The grating used in the experiment was $15 \mathrm{~mm}$ long and was created by Welltech Instrument Co. Ltd by means of exposition by a phase mask onto an UV laser beam of wavelength of $244 \mathrm{~nm}$. The FBGs written in hydrogen loaded SMF-28 fiber. It was apodized and the stripping area was $30 \mathrm{~mm}$. The central wavelength and the reflexion coefficient amounted to $1554.5 \mathrm{~nm}$ and $90 \%$ respectively. Fig. 8 presents results of the measurements of the grating reflectance spectra with variable strain. It should be noted that the width of the locking bandwidth changes: with the growing value of strain the spectral characteristics widens. The width of the spectrum in unstrained condition amounts to $0.9 \mathrm{~nm}$.

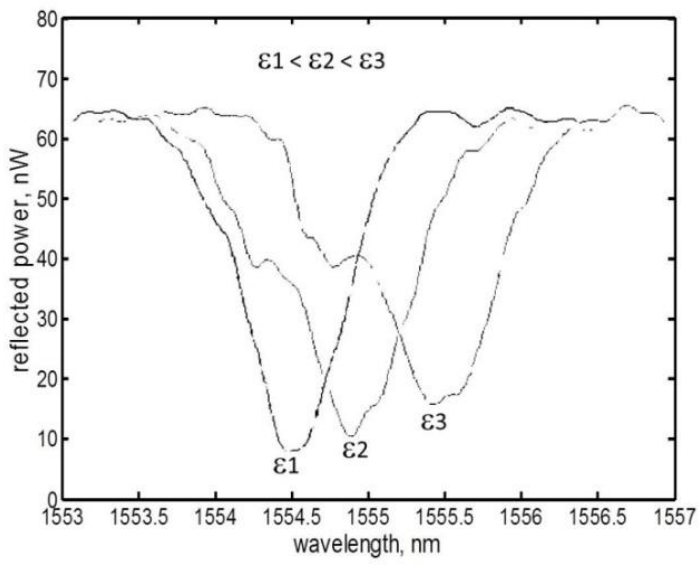

Fig. 8. Reflectance characteristics of the proposed sensor with variable strain and constant temperature.

Figure 9 presents intended FBG spectra in initial conditions $(\Delta \mathrm{T}=0)$ and with variable temperature but with constant strain. The change of temperature causes a shift of the entire 
spectral characteristics. The bandwidth remains unchanged, only the stopband of the grating is shifted. To verify their repeatability, measurements were repeated. Figs. 8-11 presents averaged results of measurements series. Additionally, no hysteresis was observed.

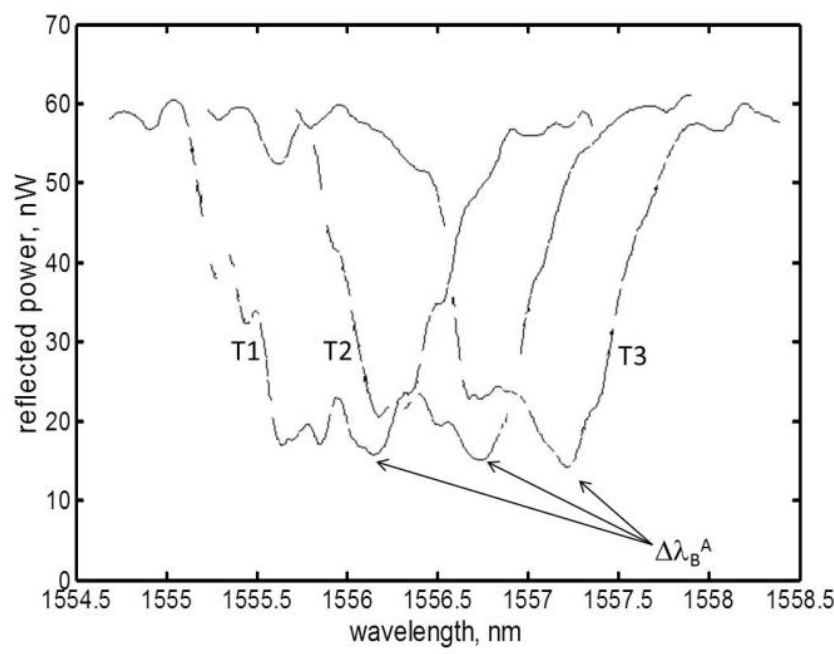

Fig. 9. Reflectance characteristics of the proposed sensor for continuous elongation and variable temperature, $\mathrm{T} 1<\mathrm{T} 2<\mathrm{T} 3$

Response of the sensor to strain, with constant temperature, is presented in Fig. 10.

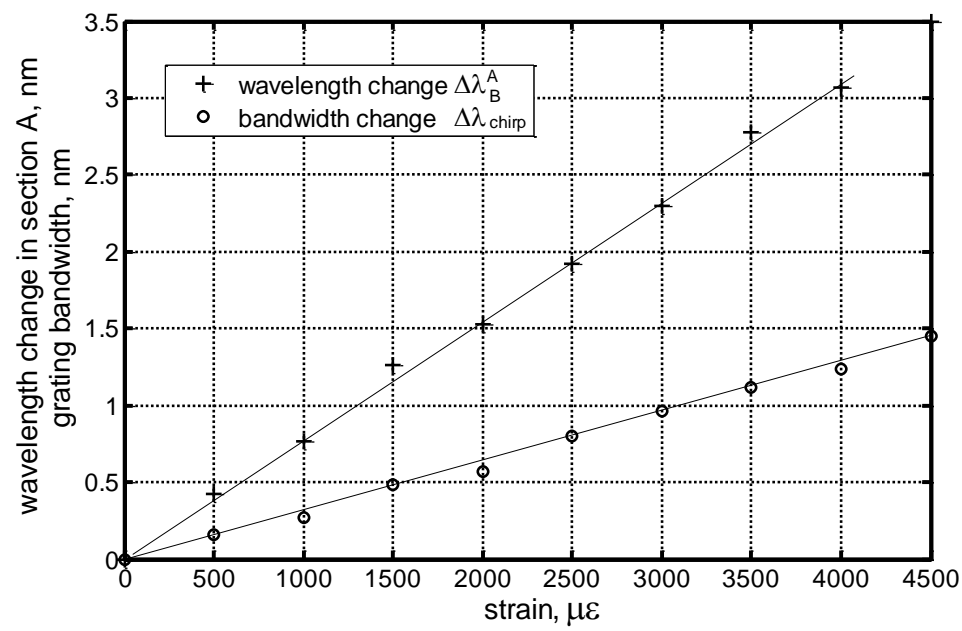

Fig. 10. Results of measurements of the sensor response to strain, obtained at a constant temperature of $25^{\circ} \mathrm{C}$.

According to theoretical analysis, especially to equation (11), the proposed sensor system shows a linear dependence of bandwidth $\Delta \lambda_{\text {chirp }}$ on the evoked strain. The Bragg wavelength shift of the grating in zone $\Delta \lambda^{A}{ }_{B}$ is also linearly dependent on strain. The regression straight lines (drawn based on the data obtained from laboratory measurements) allowed the specification of linearity of the obtained characteristics. The regression straight lines are presented in Fig. 10 with a continuous line, while nonlinearity of the characteristics of bandwidth was determined according to the volume of the non-linearity error, calculated as presented below:

$$
\delta_{\text {nlichirp }}=\frac{\Delta\left(\Delta \lambda_{\text {chirp }}\right)_{\text {MAX }}}{\left(\Delta \lambda_{\text {chirp }}\right)_{M A X}-\left(\Delta \lambda_{\text {chirp }}\right)_{M I N}} \cdot 100 \% \text {, }
$$


where $\Delta\left(\Delta \lambda_{\text {chirp }}\right)_{M A X}$ is the maximum value of absolute differences between the regression line determined from the equation, and the results of measurements of spectral width, $\left(\Delta \lambda_{\text {chirp }}\right)_{M A X}$, while $\left(\Delta \lambda_{\text {chirp }}\right)_{M I N}$ are the maximum and minimum values of the difference between reflective bandwidth of the sensor obtained from measurements, and the reflective bandwidth obtained based on linear regression. The values of nonlinearity errors, obtained in this way, amounted to $\delta_{n l \lambda c h i r p}=2.70 \%$ and $\delta_{n l \lambda A}=2.38 \%$ for spectral width $\Delta \lambda_{\text {chirp }}$ and wavelength shift $\Delta \lambda^{A}{ }_{B}$, respectively. Linear regression correlation errors amounted to 0.971 for measurements of reflective spectral width and 0.965 in the case of measurements of wavelength shift. The strain sensitivity of FBG used for testing was determined based on the angle of regression lines: $K_{\varepsilon \lambda A}=0.77 \times 10^{-6} \mathrm{~m} / \varepsilon$ and $K_{\varepsilon \lambda c h i r p}=0.31 \times 10^{-6} \mathrm{~m} / \varepsilon$.

Figure 11 presents the temperature response of the proposed sensor system. It can be seen that, with an increase of temperature, the wavelength of the reflective resonance peak increases linearly, while the full width at half maximum of the reflexive spectrum changes slightly by $\sim 0.1 \mathrm{pm}$ with an increase of temperature of $1^{\circ} \mathrm{C}$. According to mathematical analysis, the spectral characteristics of the sensor should be insensitive to temperature changes. The appearance of a slight sensitivity of the spectral width to temperature may indicate imperfection in gluing the grating to the sample, due to which there is a difference between the strain of the grating in zone A and zone B of the sample. This results in broadening of reflective spectral characteristics.

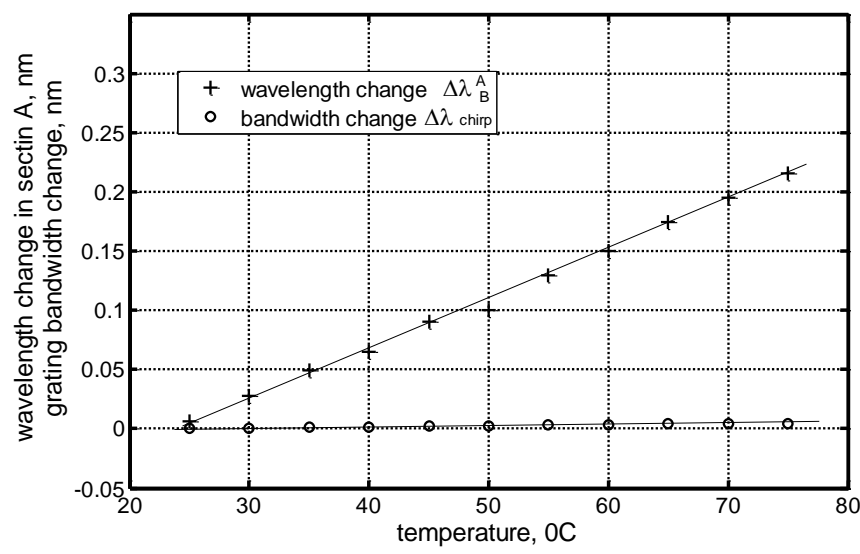

Fig. 11. The results of measurements of the sensor response to temperature, obtained at a constant strain of $1000 \mu \varepsilon$.

When measuring temperature, nonlinearity errors amounted to $1.50 \%$ and $0.01 \%$, while temperature sensitivities amounted to $K_{T \lambda A}=4.130 \times 10^{-12} \mathrm{~m} / \mathrm{K}$ and $K_{T \lambda \text { chirp }}=0.097 \times 10^{-12}$ $\mathrm{m} / \mathrm{K}$ for wavelength shift and broadening of reflective spectrum, respectively. Taking into account the sensitivities determined based on experimental measurements, creation of a new matrix (2) was possible. The equation contains current values of the designated parameters, and is now as presented below:

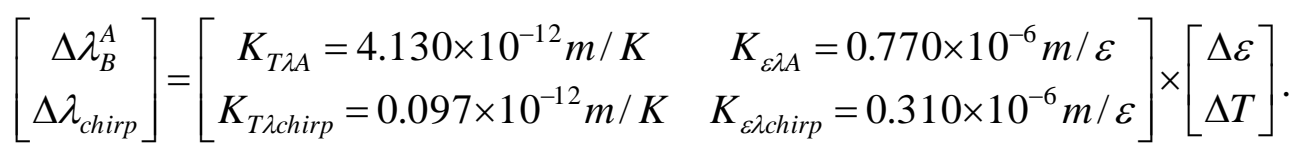

Now making a reversal of the matrix, it is possible to determine strain and temperature with the known values of the Bragg wavelength shift of the grating in zone A and the spectral width of the entire grating. The determinant of the matrix from (15) can be defined by means of the equation below:

$$
D=K_{T \lambda A} K_{\varepsilon \lambda c h i r p}-K_{\varepsilon \lambda A} K_{T \lambda c h i r p},
$$


whose value is different from zero. The good conditioning of the matrix from equation (15) allows updating of the processing (13) to the form below:

$$
\left[\begin{array}{c}
\Delta T \\
\Delta \varepsilon
\end{array}\right]=\frac{1}{1.21}\left[\begin{array}{cc}
0.311 \times 10^{6} \mathrm{~m} / \varepsilon & -0.767 \times 10^{-6} \mathrm{~m} / \varepsilon \\
-0.097 \times 10^{-12} \mathrm{~m} / \mathrm{K} & 4.129 \times 10^{-12} \mathrm{~m} / \mathrm{K}
\end{array}\right] \times\left[\begin{array}{c}
\Delta \lambda_{B}^{A} \\
\Delta \lambda_{\text {chirp }}
\end{array}\right] .
$$

Measurements of the wavelength shift and spectral width allowed an assessment of the sensor's properties. The sensor was exposed to strain from $0 \mu \varepsilon$ to $4500 \mu \varepsilon$ at a constant temperature of $40^{\circ} \mathrm{C}$ and placed in a thermal chamber with temperatures varying from $23.5^{\circ} \mathrm{C}$ to $75^{\circ} \mathrm{C}$, and with a constant strain of $2500 \mu \varepsilon$. (17) was used to determine the values of change of strain and temperature and the results of the tests and calculations are presented in Fig. 12.

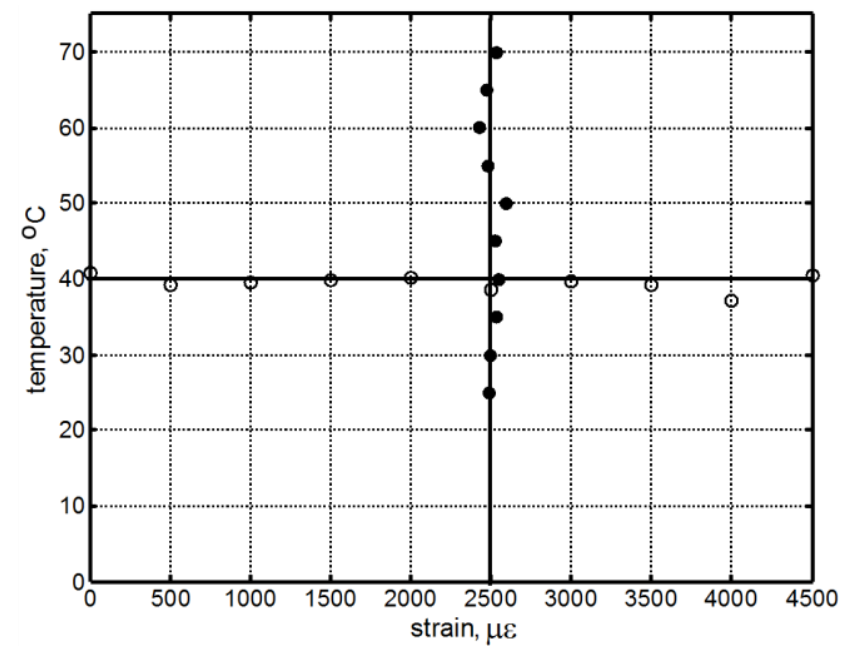

Fig. 12. Comparison of the results of parallel measurements of strain and temperature with the set values.

The straight lines in Fig. 12 denote the set values of strain and temperature, which amounted to $2500 \mu \varepsilon$ and $40^{\circ} \mathrm{C}$ respectively. When analysing Fig. 12, we can state that the results of indirect measurements of both measurands correspond with the values set during this experiment. Maximum differences between the values obtained from the direct measurement and the set points amounted to $110 \mu \varepsilon$ for strain and $3.8^{\circ} \mathrm{C}$ for temperature.

\section{Conclusions}

In conclusion, simultaneous measurement of strain and temperature, performed by means of a sensor with single uniform Bragg grating, is possible.

The obtained processing characteristics non-linearity errors for measurements of strain and temperature of the proposed system amounted to $2.7 \%$ and $1.5 \%$ respectively, while sensitivity coefficients to strain and temperature amounted to $0.77 \times 10^{-6} \mathrm{~m} / \varepsilon$ and $4.13 \times 10^{-12}$ $\mathrm{m} / \mathrm{K}$. Maximum differences between the determined and set values amounted to $110 \mu \varepsilon$ for strain and $3.8^{\circ} \mathrm{C}$ for temperature, for a strain of $2500 \mu \varepsilon$ and a temperature of $40^{\circ} \mathrm{C}$.

The disadvantage of the laboratory-tested method of simultaneous measurement of 2 measurands (strain and temperature) is the necessity of measuring the spectral width, which requires complicated systems monitoring the grating. The possibility of simultaneous measurement of strain and temperature, using a single FBG, improves the features of this type of sensor in comparison to the sensors based on several gratings or optoelectronic elements 
that modulate the spectrum with a change of the measurand. It also enables the reduction of sensor element number.

Improvement of the matrix conditions and therefore the accuracy of measurement, can be achieved by means of using adhesives of higher Young's coefficient than the epoxy adhesive used in the research process $(E \approx 2.5 \mathrm{GPa})$, (e.g. Sikadur-30 of Young's modulus $E \approx 13$ $\mathrm{GPa})$.

\section{References}

[1] Kisała, P. (2012). Metrological conditions of strain measurement optoelectronic method by the use of fibre Bragg gratings. Metrol. Meas. Syst., 19(3), 471-480.

[2] Zarnik, M.S., Belavic, D. (2012). The effect of humidity on the stability of LTCC pressure sensors. Metrol. Meas. Syst., 19(1), 133-140.

[3] Lewandowski, J. (2011). Inductive sensor for weighting of mass. Metrol. Meas. Syst., 18(2), 323-334.

[4] Xu, M.G., Archambault, J.L., Reekie, L., Dakin, J.P. (1994). Thermally-Compensated Bending Gauge Using Surface-Mounted Fibre Gratings. International Journal of Optoelectronics, 9, 281-283.

[5] Abi Kaed Bey, S.K., Sun, T., Grattan, K.T.V. (2007). Optimization of a long-period grating-based Mach Zehnder interferometer for temperature measurement. Optics Communications, 272, 15-21.

[6] Abi Kaed Bey, S.K., Sun, T., Grattan, K.T.V. (2008). Sensitivity enhancement of long period gratings for temperature measurement using the long period grating pair technique. Sensors and Actuators A, 141, 314320 .

[7] Rogers, A.J., Handerek, V.A., Kanellopoulos, S.E., Zhang, J. (1995). New ideas in nonlinear distributed optical-fiber sensing. Proc. Soc. Photo-Opt. Instrum. Eng., 2507, 162-174.

[8] Kersey, A.D., Berkoff, T.A., Morey, W.W. (1993). Fibre optic Bragg grating strain sensor with drift compensated high resolution interferometric wavelength shift detection. Optics Letters, 18(1), 72-74.

[9] Caucheteur, C., Lhomme, F., Chah, K., Blondel, M., Megret, P. (2006). Simultaneous strain and temperature sensor based on the numerical reconstruction of polarization maintaining fiber Bragg gratings. Optics and Lasers in Engineering, 44, 411-422.

[10] Lo, Y.L. (1998). Using in-fiber Bragg-grating sensors for measuring axial strain and temperature simultaneously on surfaces of structures. Optics Engineering, 37, 2272.

[11] Li, L., Tong X.L., Zhou, C.M., Wen, H.Q., Lv, D.J., Ling, K., Wen, C.S. (2011). Integration of miniature Fabry-Perot fiber optic sensor with FBG for the measurement of temperature and strain. Optics Communications, 284, 1612-1615.

[12] Wang, D., Cao, M., Li, C., Li, D., Chen, Y., Xu, X., Xu, J., Li, Y., Wan, Z., Wang, B. (2011). Fiber Bragg Grating Liquid Level Sensor with Double Pressure and Temperature Sensitivities. Procedia Engineering, 15, 704-709.

[13] Wang, T., Guo Y., Zhan, X., Zhao, M., Wang, K. (2006). Simultaneous Measurements of Strain and Temperature with Dual Fiber Bragg Gratings for Pervasive Computing. 1st International Symposium on Pervasive Computing and Applications, 786-790.

[14] Frazao, O., Romeroa, R., Araujo, F.M., Ferreira, L.A., Santos, J.L. (2005). Strain-temperature discrimination using a step spectrum profile fibre Bragg grating arrangement. Sensors and Actuators A, 120, 490-493.

[15] Li, L., Tong, X.L., Zhou, C.M., Wen, H.Q., Lv, D.J., Ling, K., Wen, C.S. (2011). Integration of miniature Fabry-Perot fiber optic sensor with FBG for the measurement of temperature and strain. Optics Communications, 284, 1612-1615.

[16] Gwandu, B.A.L, Zhang, W. (2004). Tailoring the temperature responsivity of fibre Bragg gratings. Sensors, Proc. of IEEE, 3, 1430-1433.

[17] Kisała, P. (2012). Application of inverse analysis to determine the strain distribution with optoelectronic method insensitive to temperature changes. Applied Optics, 51(16), 3599-3604. 
[18] Mroczka, J., Szczuczyński, D. (2012). Simulation research on improved regularized solution of inverse problem in spectral extinction measurements, Applied Optics, 51(11), 1715-1723.

[19] Mroczka, J., Szczuczyński, D. (2009). Inverse problems formulated in terms of first-kind Fredholm integral equations in indirect measurements. Metrol. Meas. Syst., 16(3), 333-357.

[20] Mroczka, J., Szczuczyński, D. (2010). Improved regularized solution of the inverse problem in turbidimetric measurements, Applied Optics, 49(24), 4591-4603. 